"The wronk dlescribed in this thesis was carmiedl out by me wander the

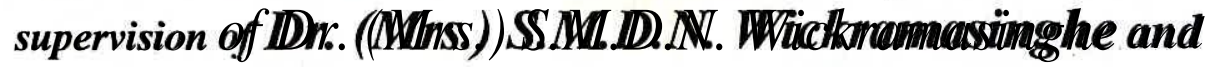
Professor (Mrs.) P. Angunawela and a report on this has not been submitted to any University for another degree ${ }^{m}$

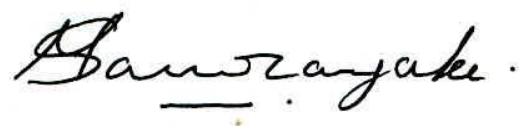

Mrs. M.D.P. Samaranayake. 
"We certify that the above statement made by the candidate is true and that this thesis is suitable for submission to the university for the purpose of evaluation"

S.M.N. Wickramas ift

Dr. (Mrs.) S.M.D.N. Wickramasinghe.

Senior Lecturer,

Dept. of Biochemistry,

Faculty of Medical Sciences,

Uni. of Sri Jayawardenepura.

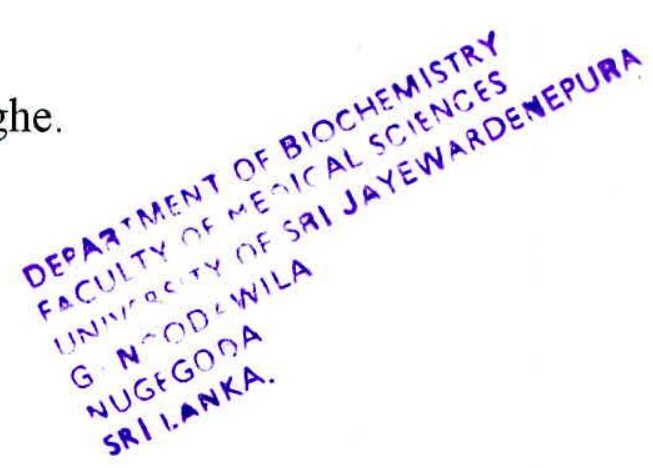

$\frac{\text { P. Anguma wohl }}{\text { Prof. (Mrs.) P. Angunawela, }}$

Senior Lecturer,

Dept. of Pathology,

Faculty of Medicine,

Uni. of Colombo. 


\section{Effects of garlic (Allium sativum) on chemically- induced hepatocarcinogenesis in normal and hypercholesterolaemic Wistar rats}

\section{Mala Damayanthi Perera Samaranayake}

Thesis submitted to the University of Sri Jayawardenepura for the award of the degree of Master of Philosophy in Biochemistry on $05^{\text {th }}$ June 2000. 


\section{CONTENTS}

Table of contents

i-vii

List of Tables

viii-ix

List of Figures

$\mathbf{x}$

List of Plates

$\mathbf{x i}$

ACKNOWLEDGEMENTS

xii-xiv

ABSTRACT

xv-xvi

\section{INTRODUCTION}

1.1 Background and justification

1.2 Scope of the study

\section{LITERATURE REVIEW}

2.1.1 Botanical aspects 6

2.1.2 Major Chemical constituents 6-7

2.1.3 Medicinal and other uses

2.1.3.1 Introduction 8

2.1.3.2 Carminative and stimulative properties 8

2.1.3.3 Expectorant properties 9

2.1.3.4 Hypolipaemic and hypocholesterolaemic

Properties 9

2.1.3.5 Anti-thrombotic properties 9

2.1.3.6 Anti-hypertensive properties 9

$\begin{array}{lll}\text { 2.1.3.7 Anti-microbial properties } & 10\end{array}$ 
2.1.3.8 Anthelmintic properties

2.1.3.9 Anti-turberculosis properties

2.1.3.10 Antioxidative properties

2.1.3.11 Hypoglycaemic properties

2.1.3.12 Malignant diseases

2.1.3.13 Other uses in medicine

\subsection{Carcinogenesis}

2.2.1 Introduction to carcinogenesis

2.2.2 Carcinogen

2.2.2.1 Chemical carcinogen

2.1.2.2 Physical carcinogen

2.1.2.3 Biological carcinogen

2.2.3 Mechanism of carcinogenesis

2.2.3.1 Two step carcinogenesis

2.2.3.2 Multi stage carcinogenesis

2.2.4 Dietary fat and carcinogenesis

2.2.4.1 Introduction

2.2.4.2 Hepatocarcinogenesis

\subsection{Tumour markers}

2.3.1 Definition

2.3.2 Features of a tumour marker

2.3.3 Classification

2.3.3.1 Enzymes

2.3.3.1.1 Introduction 


\subsection{Gluutathione S-transferase (GST)}

2.3.3.1.2.1 Introduction 23

2.3.3.1.2.2 Classification 23-24

2.3.3.1.2.3 Structure 24

2.3.3.1.2.4 Functions 24

2.3.3.1.2.1 As a tumour marker 24-26

2.3.3.1.2.4.2 Detoxification Properties 26

2.3.3.1.4.3 Other functions $\quad 26$

2.3.3.2 Oncofetal antigen

2.3.3.2.1 Introduction

2.3.3.3 Hormones and Hormone Receptors

2.3.3.3.1 Introduction

2.3.3.4 Carbohydrate Markers

2.3.3.4.1 Introduction

2.3.3.5 Genetic markers

2.3.3.5.1 Introduction $29-30$

2.3.3.5.2 Oncogenes

2.3.3.5.3 Suppressor genes 


\section{MATERIALS AND METHODS}

\subsection{Materials}

3.1.1 Plant Material 31

3.1.2 Animal Model 31

3.1.3 Chemicals 31

\subsection{Methods}

3.2.1 Development of a hypercholesterolaemic rat model

3.2.1.1 Collection of blood samples and separation of serum

3.2.1.2 Quantitative determination of total serum cholesterol

3.2.1.2.1 Preparation of cholesterol reagent

3.2.1.2.2 Procedure

3.2.2 Liver Medium-Term Bioassay Protocol

3.2.2.1 Experimental design

3.2.2.2 Partial hepatectomy

3.2.2.2.1 Procedure

3.2.2.3 Collection and fixation of liver tissues

3.2.2.3.1 Preparation of $10 \%$ buffered formalin

3.2.2.4 Processing, embedding and cutting of liver tissues

3.2.2.5 Gluutathione S-transferase-P (GST-P) immunohistochemistry 
3.2.2.5.2 Preparation of phosphate buffered saline(PBS)

3.2.2.6 Haematoxylin \& Eosin staining

3.2.2.6.1 Procedure

3.2.2.7 Histopathological examination

3.2.2.8 Dosage and administration of garlic (Allium sativum)

3.2.2.9 Induction of carcinogenicity

3.2.2.10 Statistical analysis

\subsection{Experimental}

3.3.1 Development of a Hypercholesterolaemic rat model

3.3.1.1 Experiment I; Effects of cholesterol-enriched diets $(0.25 \%, 0.5 \%, \& 1 \%)$ on the level of serum cholesterol in male Wistar rats.

3.3.1.2 Experiment II; Effects of treatment with $0.5 \%$ cholesterol-enriched diet for six weeks on level of serum cholesterol in male Wistar rats.

3.3.2 Effects of garlic on chemically-induced hepatocarcinogenesis in male Wistar rats.

3.3.2.1 Experiment III: Effects of garlic on chemicallyinduced hepatocarcinogenesis in normal Wistar rats.

3.3.2.1.1 Animal experiment

3.3.2.2 Experiment IV: Effects of garlic treatment on Chemically-induced hepatocarcinogenesis in hypercholesterolaemic male Wistar rats 


\section{RESULTS}

4.1 Development of a hypercholesterolaemic rat model

4.1.1 Effects of cholesterol-enriched diets

$$
(0.25 \%, 0.5 \% \& 1 \%)
$$

4.1.1.1 On the serum cholesterol level

4.1.1.2 On average body weight

4.1.2 Effects of treatment with $0.5 \%$ cholesterolenriched diet for six weeks on the serum cholesterol level

4.1.2.1 On the of serum cholesterol level

4.1.2.2 On average body weight

4.2 Effects of garlic on DEN-induced hepatocarcinogenesis in normal Wistar rats

4.2.1 Inhibition of glutathione s-transferase placental form positive $\left(\mathrm{GST}^{-} \mathrm{P}^{+}\right)$foci

4.2.2 Histopathological evidence

4.2.3 Body weights 58,62

4.3 Effects of garlic on DEN-induced hepatocarcinogenesis in hypercholesterolaemic Wistar rats

4.3.1 Inhibition of GST-P ${ }^{+}$foci induction

4.3.2 Histopathological evidence

4.3.3 Body weights 70-70

4.3.4 Level of mean total serum cholesterol 70,72

4.4 Comparison of GST-P ${ }^{+}$foci induction in normal and hypercholesterolaemic Wistar rats

4.4.1 Number and area of GST-P $\mathrm{P}^{+}$foci induction 
5. DISCUSSION

6. CONCLUSIONS

7. SUGGESIONS FOR FURTHER STUDIES

8. REFERENCES 


\section{LIST OF TABLES}

Table 2.1 Enzymes as tumor Markers

Table 2.2 Oncofetal antigens as tumor Markers

Table 2.3 Hormones and Hormone receptors as tumor Markers

Table 2.4 Carbohydrate markers as tumor Markers

Table 2.5 Genetic markers as tumor Markers

Table 3.1 Feed formulae for standard rat feed

Table 4.1 Effects of cholesterol-enriched diets containing different amounts of cholesterol on level of serum cholesterol in Wistar rats

Table 4.2 Effects of cholesterol-enriched diets on mean body weights of Wistar rats

Tabl: 4.3 Effects of treatment of $0.5 \%$ cholesterol-enriched diets for six week on the mean body weights of Wistar rats for six weeks

Table 4.4 Effects of Garlic on GST-P $\mathrm{P}^{+}$liver foci in DEN-induced hepatocarcinogenesis in normal Wistar rats.

Table 4.5 Effects of Garlic treatment on the histopathology of liver sections of normal Wistar rats

Table 4.6 Effects of Garlic on mean body weights of normal Wistar rats in DEN-induced hepatocarcinogenesis 
Table 4.7 Effects of Garlic on GST-P ${ }^{+}$liver foci in DEN-induced hepatocarcinogenesis in hypercholesterolaemic Wistar Rats

Table 4.8 Effects of Garlic treatment on histopathology of liver sections of hypercholesterolaemic Wistar rats

Table 4.9 Effects of Garlic on mean body weights of hypercholesterolaemic Wistar rats in DEN-induced hepatocarcinogenesis

Table 4.10 Effects of Garlic on the level of serum total cholesterol in hypercholesterolaemic Wistar rats 


\section{LIST OF FIGURES}

Figure 3.1 Standard curve for serum total cholesterol

Figure 3.2 Assay Method (Liver Medium-term bio assay

Protocol)

Figure 3.3 Assay Method (Modified Liver Medium-term bio assay

Protocol)

Figure 4.1 Effects of $0.5 \%$ cholesterol-enriched diet

on the level of serum cholesterol in Wistar rats.

Figure 4.2 Effects of cholesterol-enriched diet on number

of DEN-induced liver GST- $\mathrm{P}^{+}$foci in Wistar rats

Figure 4.3 Effects of cholesterol-enriched diet on area

of DEN-induced liver GST- $\mathrm{P}^{+}$foci in Wistar rats 


\section{LIST OF PLATES}

Plate 2.1 Bulbs of Garlic (Allium sativum)

Plate 2.2 The Crab and Cancer

Plate 3.1 Procedure of partial hepatectomy in rat.

$\left(A_{3.1}-G_{3.1}\right)$

Plate 4.1 Immunohistochemical Staining of DEN-induced glutathione S-transferase placental form $\left(\right.$ GST-P ${ }^{+}$) foci, (haematoxylene counterstain) in normal Wistar rats. $\left(\mathrm{A}_{4.1}, \mathrm{~B}_{4.1}\right)$

Plate 4.2 Representative microphotographs showing the effects of garlic treatment on histopathalogy of liver tissues of normal Wistar rats.

$\left(\mathrm{A}_{4.2}-\mathrm{C}_{4.2}\right)$

Plate 4.3 Immunohistochemical Staining of DEN-induced glutathione S-transferase placental form positive $\left(\right.$ GST-P ${ }^{+}$) foci, (haematoxylene counterstain) in hypercholesterolaemic Wistar rats. $\left(\mathrm{a}_{4.3}, \mathrm{~b}_{4.3}\right)$

Plate 4.4 Representative microphotographs showing the effects of garlic treatment on histopathalogy of liver tissues of hypercholesterolaemic Wistar rats. $\left(\mathrm{a}_{4.4}-\mathrm{c}_{4.4}\right)$ 


\section{AKNOWLEDGEMENTS}

M y senior supervisor, Dr. (Mrs.) S.M.D.N. Wickramasinghe, Senior Lecturer, Dept of Biochemistry, Faculty of Medical Sciences, University of Sri JayEwardenepura, is greatly acknowledged for introducing me into the field of cancer research and for her valuable advice, encouragement, friendly supervision and creating a stressless research environment .

I wish to express my foremost appreciation to my co-supervisor Prof. (Mrs.) P. Angunawela, Senior lecturer, Dept of Pathology, Faculty of Medicine, University of Colombo who supervised me, and made arrangements to get an exposure to the field of histopathology and immunohistochemistry.

My grateful thanks to Dr. (Mrs.) S. Jayasekara, Head/ Animal center, Medical Research Institute, Sri Lanka, who was my un-official supervisor, trained me in all aspects of rat handling and also for her ever-ready support throughout this study. It should be mentioned that this research would not have been made possible if her support and facilities in animal center were not made available.

My grateful thanks to Prof. E. R, Jansz, Professor and former Head, Dept of Biochemistry, Faculty of Medical Sciences, University of Sri Jayawardenepura , Sri Lanka for introducing me into the field of Biochemistry, providing me the carcinogen (diethylnitrosoamine) to initiate the study at the initial stages and for allowing me to use all available facilities at the department of Biochemistry. 
Thanks are also due to Dr (Miss) M. Tammitiyagoda, Veterinary Surgeon, Animal Center, Medical Research Institute (M.R.I.), Sri Lanka, for her valuable support specially during animal surgery, collecting samples, in training of animal handling and also for memorable happy times, snacks, tea etc. I must thank Mr.S. S. Sisira Kumara and Mr. Jayasiri ,Animal Center, M.R.I. for their assistance and all kinds of help given me at the M.R.I., Sri Lanka.

My grateful thanks are also due to Prof. S. Fukushima, Professor, Department of Pathology, Osaka City University, Japan, for providing the expensive glutathione Stransferase antibody free of charge and literature and information related to immunohistochemical assay method.

I wish to thank Dr. S. Iwai, Department of Pathology, Osaka City University, Japan, for reading immunohistological tissue sections.

Thanks are also due to Prof. (Mrs.) C. Pathirana, Head/ Department of Biochemistry, Faculty of Medicine, University of Ruhuna, Sri Lanka, for her encouragement and allowing me to use departmental facilities in preparing this thesis and Dr.(Miss.) $\mathrm{K}$. Jayathilake, senior lecturer Department of Biochemistry, Faculty of Medicine, University of Ruhuna, Sri Lanka, for her valuable comments and encouragement in making this thesis a success. 
I wish to thank Dr (Mrs.) M.V. Werasooriya, Head/ Department of Parasitology, Faculty of Medicine, University of Ruhuna for giving me permission to use research microscope in taking microphotographs. Also I would like to thank Miss. M. Mudalige, Department of Parasitology, Faculty of Medicine, University of Ruhuna, for her everready help in taking microphotographs.

My gratitude also goes to Oushada Lanka (pvt.) Ltd., Katuwawala, Sri Lanka for providing garlic powder.

My thanks also go to Mrs S. Ekanayake, Department of Biochemistry, Faculty of Medical sciences, University of Sri Jayawardenepura, Sri Lanka for her comments in preparing this thesis.

Staff of the Department of Biochemistry, Faculty of Medical sciences, University of Sri Jayawardenepura and laboratory staff of the Department of Pathology, Faculty of Medicine, University of Colombo, Sri Lanka are greatly appreciated for their assistance during this study.

Mr. H. Pathirana is acknowledged for his valuable assistance in making computer settings of the thesis.

Finally, I wish to thank my husband for his ever- ready support and my dear son who had to spend his infancy with less attention and love from me. 


\section{ABSTRACT}

Hepatocellular carcinoma is one of the most common cancers in the modern world and it is prevalent in Asia. Environmental and dietary factors play an major role in the development of many cancers. Dietary modification can also play important role in reducing the risk of cancer. Garlic (Allium sativum) is a well known medicinal herb and a food item that has been used all over the world since pre historic times. Previous studies using cancer cell lines and animal models have indicated that garlic and some of its sulfur compounds are potential anticarcinogens.

The present study was carried out to investigate the effects of a therapeutic dose (20 $\mathrm{mg} / \mathrm{kg}$ body wt./day) of garlic on chemically-induced hepatocarcinogenesis in normal and hypercholesterolaemic Wistar rats. In the fist phase of this study the inhibitory effects of a therapeutic dose $(20 \mathrm{mg} / \mathrm{kg}$ body wt./day) of garlic on diethylnitrosamine (DEN)- induced neoplasia of the liver were examined in normal male Wistar rats. Medium-term Bio assay system of Ito based on the two step model of hepatocarcinogenesis was used as the assay method.

In phase II, the effects of garlic on hepatocarcinogenesis in hypercholesterolaemic Wistar rats were investigated. The selectiuon of hypercholesterolaemic model was based on the available evidence for the relationship between dietary fat and the development of cancer. Hypercholesterolaemic model was developed by feeding rats with a $0.5 \%$ cholesterol-enriched diet for a period of two weeks. Rats having serum cholesterol level $>120 \mathrm{mg} / \mathrm{dl}$. were considered as hypercholesterolaemic ( normal range $75 \pm 10 \mathrm{mg} / \mathrm{dl}$ ) and the cholesterol-enriched diet was continued throughout the 
experiment. Carcinogenic potential was scored by comparing the number and area of induced Glutathione S-transferase placental form positive (GST- $\mathrm{P}^{+}$) liver foci as well as histopathological examination of liver sections.

Daily treatment with garlic markedly reduced the number and area of GST-P ${ }^{+}$foci (48\% inhibition and $49 \%$ inhibition respectively) as compared with the control group of animals receiving distilled water. Significant inhibition of induction of GST-P ${ }^{+}$foci ( $34 \%$ inhibition in number and $44 \%$ inhibition in area ) due to garlic treatment was also observed in the hypercholesterolaemic group of rats. However percentage inhibition was higher in garlic treated normal rats than garlic treated hypercholesterolaemic rats. Also it was evident from this study that hypercholesterolaemic rats are more susceptible to induction of liver GST- $\mathrm{P}^{+}$foci.

Results of GST-P ${ }^{+}$expression were supplemented by histopathological examination of liver sections of garlic treated normal as well as hypercholesterolaemic Wistar rats. Granular and vacuolar degeneration were used as parameters in assessing histopathological alterations. In normal rats, garlic treatment reduced the pathological alterations in liver sections caused by DEN. Similar results were obtained from experiments with hypercholesterolaemic rats.

Hence the results of this study provide strong supportive evidence for the anticarcinogenic activity of garlic. 